\title{
COLLAPSE OF WHITE DWARFS \\ IN CLOSE BINARY SYSTEMS
}

R. Canal

and

$\mathrm{J}$. Isern

Departamento de Fisica

de la Tierra y del Cosmos

Universidad de Barcelona

The presence of neutron stars in close binary systems, shown by the pulsating $X$-ray sources, poses the problem of their origin. In the case of the low-mass $\left(M_{1}+M_{2} \leq 5 M_{0}\right) X$-ray binaries, the neutron star might have originated from a massive white dwarf, driven over the Chandrasekhar 11mit by mass transfer (Schatzman 1974). A similar scenario had been put forward by Whelan and Iben (1973) for type I supernovae. To solve the problem of the very low eccentricities observed for the orbits, and to facilitate keeping the system bound after neutron star formation, Canal and Schatzman (1976) suggested a non explosive collapse of the white dwarf to a neutron star. The occurence of this kind of collapse depended on the possibility of avolding thermonuclear ignition by means of neutronization. Since there is a density interval where the electron captures on carbon go faster than the pycnonuclear reactions, just above the critical density for the beginning of the collapse, there seemed also to be a chance of escape from thermonuclear runaway. A closer examination of this picture leads, however, to significant changes.

Let us assume that the mass transfer begins on a carbon-oxygen white dwarf, as close as possible to the dynamical instabiliffy limit.

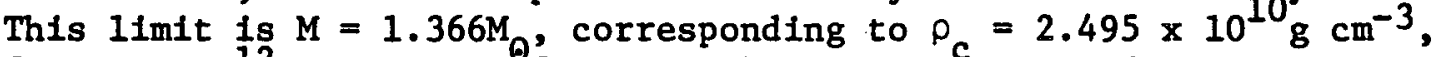
for a pure ${ }^{12} \mathrm{C}$ white dwarf, where the instability is due to general-relativistic effects. It is $\mathrm{M}=1.365 \mathrm{M}$, corresponding to $\rho_{c}=1.920 \times 10^{10} \mathrm{~g} \mathrm{~cm}^{-3}$, for a ${ }^{12} \mathrm{c}-160$ white dwarf, where the collapse is first induced by the electron caputres on oxygen. How close to these limits a stable ${ }^{12} \mathrm{C}-160$ degenerate star can be is determined by the balance between energy generation by the ${ }^{12} \mathrm{C}+{ }^{12} \mathrm{C}$ reactions (minus the neutrino losses) and conductive heat transport. That gives $M \simeq$ $1.357 \mathrm{M}_{\theta}\left(\rho_{c} \simeq 6 \times 10^{9} \mathrm{~g} \mathrm{~cm}^{-3}\right)$ for a pure carbon white dwarf.

We further assume an accretion rate equal to the Eddington 11mit. In order to avold thermonuclear runaway at the star's center, prior to the collapse, we must have $\tau_{0}<\tau_{\text {th }}$, where $\tau_{0}$ and $\tau$ th are the time scales for the increase in central density ahd for thiclear 
heating, respectively. For low $\left(\mathrm{T}_{\mathrm{c}} \leqq 10^{7} \mathrm{~K}\right)$ initial internal temperatures we find that a pure carbon white dwarf woyld ignite at a central density, $\rho_{c}$, only slightly above $10^{10} \mathrm{~g} \mathrm{~cm}^{-3}$. This is illustrated in Figure 1, for the pycnonuclear rates of Salpeter and Van Horn (1969). The result depends, of course, on the accretion and nuclear reaction rates. An improved treatment of the short-range behavior of the quantum-mechanical pair correlation function for a strongly coupled plasma (Schatzman 1978; Alastuey and Jancovic1 1979) do very significantly increase. $\tau$. But it does not yet cover the entire range of temperatures and densities which are relevant to our problem. Different ways for violating the classical Eddington limit could produce the same effect: to reach the dynamical instability line before ignition. But even in this case, when $\rho_{c} \geq 3.49 \mathrm{x}$ $10^{10} \mathrm{~g} \mathrm{~cm}^{-3}$ the electron captures on ${ }^{12} \mathrm{C}$ start. Their thermal effect (Bruenn 1973) is to contribute to the temperature increase, together with compression and the pycnonuclear reactions. This results in thermonuclear runaway well before exhaustion of the ${ }^{12} \mathrm{C}$ fuel by the electron captures, but when the star is already collapsing and the density is high enough to insure the formation of a bound remant after incineration (Bruenn 1972; Mazurek et al. 1974).

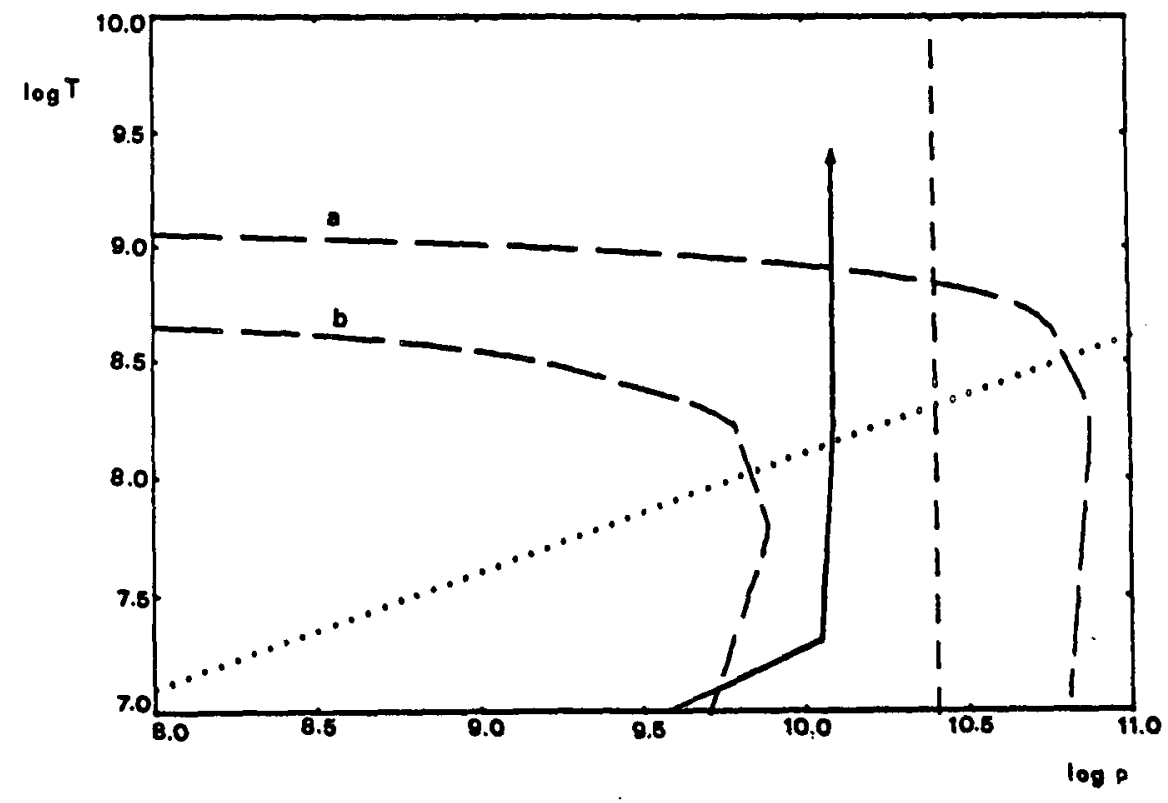

Fig. 1 - Evolution of a pure carbon white dwarf's center for mass accretion at the Eddington limit. Dashed curve a is the limit for explosive burning. $\underline{b}$, that for thermal stability. Vertical dashed line is the dynamical stability limit. Dotted line separates crystallization and Coulomb liquid states. 
We consider now the more realistic case of a ${ }^{12} \mathrm{C}-{ }^{16} 0$ whyte dwarf. The dynamical instability arises from electron captures on 160 when the mass fraction of this nuclide surpasses $6 \%$. Reaching that point before thermonuclear runaway becomes easier with increasing fractions of ${ }^{16} \mathrm{O}$. But, as for the captures on ${ }^{12} \mathrm{C}$, those on ${ }^{16} \mathrm{O}$ do accelerate the star's ignition.

So we have, in all cases, that a thermonuclear runaway develops at the center of the star, with $\rho_{c} \geqslant 10^{10} \mathrm{~g} \mathrm{~cm}^{-3}$. When this happens, the dynamical condition of the white dwarf can be either quasi-static contraction or collapse, depending on the uncertainties in chemical composition and in the accretion and thermonuclear reaction rates.

The subsequent evolution is mainly determined by the regime of combustion of the nuclear fuel. If a detonation wave were initlated at the star's center, it would self-consistently propagate. That would lead to total disruption of the star if it happened in the quasi-static phase, or leave a bound remnant, had the collapse already started. The formation of such a wave is not likely, however. Mazurek et al. (1977) have shown, on energetic grounds, that a detonation cannot form upon carbon ignition for $\rho \gtrsim 10^{3} \mathrm{~g} \mathrm{~cm}^{-3}$. Spherical damping, as discussed by ono (1960), acts to the same effect (Nomoto et al. 1976). So, we adopt a deflagrative regime for the carbon-oxygen burning.

Our preliminary results show that carbon deflagration leads to the gravitational collapse of the star, due to the electron captures on the incinerated material. That differs from Nomoto et al. (1976), who obtained a complete disruption of their degenerate carbon-oxygen cores through deflagration. The difference mainly arises from the fact that the present calculations start from much higher central densities and lower internal temperatures, where most of the star's mass is on the crystallization zone of the temperature-density diagram. There, the burning propagates only by conduction. The corresponding velocities are several orders of magnitude smaller than those associated with the convective deflagration. So, our results are similar to those of the calculations reported by Chechetkin et al. (1977), where no mechanisms of propagation of burning are included but hydrodynamical compression. We cannot yet ascertain whether a fraction of the star's mass can be expelled or not.

The whole viability of the previous scheme depends, of course, on the accreted matter belng actually incorporated to the star. Approximate calculations of the effects of inflow of hydrogen-rich material on the carbon-oxygen white dwarf have been made, based on the scheme of Saslaw (1968). They give that, for accretion at the Eddington limit, a $1.3 \mathrm{M}_{\theta}$ star with internal temperature $T \leqslant 10^{7} \mathrm{~K}$ would deflagrate its center betore flashing its accreted layer. But more refined model calculations are needed before we can set reliable limits to the rate of Increase in central density and to determine the exact configuration of the core when deflagration begins. 
We conclude that the non-explosive collapse of a massive white dwarf to a neutron star is likely, the range of initlal conditions leading to it remaining to be further explored. Were the outer layers in some cases ejected, disruption of the system might be one possible origin for pulsars.

\section{REFERENCES}

Alastuey, A., and Jancovic1, B. 1978, Ap.J., 226, 1034. Bruenn, S.W. 1972, Ap.J. Supp1., 24, 283.

-1 1973, Ap.J. Lett., 183, L125.

Canal, R., and Schatzman, E. 1976, Astron. and Astrophys., 46, 229. Chechetkin, V.M., Imshennik, V.S., Ivanova, L.N., and Nadyozhin, D.K. 1977, in Supernovae, ed. D.N. Schramm (Reidel: Dordrecht), p.159.

Mazurek, T.J., Truran, J.W., and Cameron, A.G.W. 1974, Astrophys. and Space Sc1., 27, 261.

Mazurek, T.J., Meier, D.L., and Wheeler, J.C. 1977, Ap.J., 213, 518.

Nomoto, K., Sugimoto, D., and Neo, S. 1976, Astrophys. and Space Sci., 39, L37.

Ono, Y. 1960, Progr. Theor. Phys., Kyoto, 24, 825.

Salpeter, E.E., and Van Horn, H.M. 1969, Ap.J., 155, 183.

Saslaw, W. 1968, M.N.R.A.S., 138, 337.

Schatzman, E. 1974, in The Nuclei of Galaxies, Black Holes, and Collapsed Matter, Course III, Inter. School of Cosmology and Gravitation, Erice, Italy.

- 1978, Astron. and Astrophys., 65, L17.

Whelan, J., and Iben, I. 1973, Ap.J., 186, 1007. 